\title{
A Model of Mitochondria in the Rat Hepatocyte
}

\author{
William Bell ${ }^{1}$ and Anita T. Layton ${ }^{2}$
}

1. Department of Applied Mathematics, University of Waterloo, Waterloo, Ontario, Canada

2. Departments of Applied Mathematics and Biology, Cheriton School of Computer Science, School of Pharmacy, University of Waterloo, Waterloo, Ontario, Canada 


\begin{abstract}
Mitochondria are a key player in several kinds of tissue injury, and are even the ultimate cause of certain diseases. In this work we introduce a new model of mitochondrial ATP generation in liver hepatocytes of the rat. Ischemia-reperfusion is an intriguing example of a non-equilibrium behaviour driven by a change in tissue oxygen tension. Ischemia involves prolonged hypoxia, followed by the sudden return of oxygen during reperfusion. During reperfusion, we predict that the build up of succinate causes the electron transport chain in the liver to temporarily be in a highly reduced state. This can lead to the production of reactive oxygen species. We accurately predict the timescale on which the electron transport chain is left in a reduced state, and we observe levels of reduction likely to lead to reactive oxygen species production. Aside from the above, we predict thresholds for ATP depletion from hypoxia, and we predict the consequences for oxygen consumption of uncoupling.
\end{abstract}




\section{Introduction}

Mitochondria are crucial to understanding the pathophysiology of the liver in a wide array of circumstances. Some of the ways in which liver pathophysiology specifically is impacted by or mediated through mitochondria are not unique to the liver. Hypoxia is a common consequence of liver disease [55] and ischemia-reperfusion injury is a common consequence of liver transplantation [41] but they are certainly not unique to the liver. Other mitochondriarelated pathophysiologies are unique to the liver. For instance alcoholic fatty liver disease is of course unique to the liver, and mitochondria are causally-connected to its development [61]. These unique problems for the liver also are associated with special adaptive responses. For instance during alcohol consumption, in healthy rats there is a large increase in liver oxygen tension [47]. This could potentially prevent hypoxia but could also increase oxidative stress, which is associated with alcohol-related liver injury [61]. In light of all these conditions, understanding mitochondria is crucial to understanding liver pathophysiology, but it is also necessary that the liver is recognized as the unique organ that it is when investigating mitochondria-related liver pathophysiology.

Since our interest is in a cellular organelle, we take a subcellular modelling approach to study this system. We study a system of ordinary differential equations which describe pyruvate oxidation, the tricarboxylic acid (TCA) cycle, and oxidative phosphorylation (OXPHOS). We take a model originally developed for cardiac muscle tissue [60], and since adapted to study the proximal tubule in the kidney [14], and adapt it into a model for hepatocytes. We opt to preferentially study hepatocytes within the liver because by volume the liver is composed of $80 \%$ hepatocytes [3]. The second-most common cell type, stellate cells, make up only $5-8 \%$ of liver cells [18]. Hepatocytes are also are the basis for many critical liver functions, including those related to energy metabolism, such as glycogenesis and gluconeogenesis $[42]$.

We aim to develop a mathematical model of the hepatocyte mitochondrial function in both healthy and pathological conditions. Under baseline (healthy) conditions, we need to consider a range of values both to account for uncertainty and to account for heterogeneity between hepatocytes [4]. For our stated purpose of studying mitochondrial function, gradients of oxygen and preferences of metabolic pathways within liver lobules are particularly important. Other quantities are highly uncertain and must be varied for that reason. In particular ATP consumption and the contribution of glycolysis to liver function (the latter is also subject to spatial heterogeneity). When we examine the sensitivity of our model under health conditions, we'll be interested especially to identify the sensitivity of the default conditions to all the features mentioned here.

We then apply the model to investigate several causes of liver dysfunction, namely: mitochondrial disease (and especially those based on OXPHOS-mediated disorders), fatty liver disease (which especially causes oxidative stress, leading to OXPHOS dysfunction and Cytochrome $\mathrm{C}$ release), hypoxia, and acute alcohol consumption (which causes oxidative stress). Crucially, many of these either lead to or follow from OXPHOS dysfunction, and so our aim will be two-fold: to replicate conditions of a highly reduced OXPHOS chain, the natural 
preceding event to oxidative stress, and to consider OXPHOS dysfunction once it occurs.

\section{Method}

\subsection{The Model}

The model is adapted from a model for cells in the kidney of the rat. The biochemical reactions and transport kinetics in the two tissues are relatively similar, and so the model structure isn't significantly altered for this work. The only structural change to the kidney model is the introduction of glutamate dehydrogenase activity, described below. The key changes relative to either the proximal tubule (PT) or medullary thick ascending limb (mTAL) model were to the parameters, including some clamped states of the model. This happened in two phases: first we changed parameters that had been measured experimentally, and second we fitted some less measurable parameters. Section 2.2 addresses this second fitting phase. In this section we address experimentally determined parameter values.

\begin{tabular}{|c|c|c|}
\hline Parameter & Values for Liver & Reference \\
\hline Fractional Mitochondrial Volume (L mito/L cell) & 0.23 & {$[3]$} \\
\hline Fractional Cytoplasmic Volume (L cyto/L cell) & 0.70 & {$[3]$} \\
\hline Cytoplasm Water Fraction (L water/L cyto) & 0.77 & [53] \\
\hline Mitochondria Water Fraction (L water/L mito) & 0.58 & {$[54]$} \\
\hline Matrix NAD+NADH concentration (mmol/L matrix) & $9.4 \times 10^{-3}$ & {$[8]$} \\
\hline Matrix concentration of co-enzyme Q (mmol/L matrix) & $6.49 \times 10^{-3}$ & {$[5]$} \\
\hline Intermembrane concentration of Cytochrome $\mathrm{C}(\mathrm{mmol} / \mathrm{L} \mathrm{IM})$ & $4.39 \times 10^{-3}$ & {$[5]$} \\
\hline Protein Density of Mitochondria (mg prot/L mito) & $6.45 \times 10^{5}$ & [29] \\
\hline Liver-to-heart Ratio Complex I & 1.2 & {$[5]$} \\
\hline Liver-to-heart Ratio Complex III & 0.525 & {$[5]$} \\
\hline Liver-to-heart Ratio Complex IV & 0.40 & {$[5]$} \\
\hline Mitochondrial Oxygen Tension (mmHg) & 35 & {$[39]$} \\
\hline Cytosolic Potassium Concentration (mmol/L cyto) & 88 & {$[57]$} \\
\hline
\end{tabular}

Table 1: A list of parameters used in our model that were based on experimental data.

Several important quantities for the model can be measured directly, they are listed in Table 1. ATP consumption ranges were chosen based on the activity of Na-K-ATPase in mice [25] and an estimate in rats that $20 \%$ of ATP consumption (via a proxy of oxygen consumption) is due to Na-K-ATPase [2]. This gave us a reasonable-appearing estimate that the maximal ATP consumption was roughly half of that of a proximal tubule cell in the kidney. Proximal tubule cells have particularly large energy requirements, suggesting this is reasonable [14]. Glycolysis-based ATP production was taken to be a third of the maximal glycolysis-mediated pyruvate and lactate production found in rats in Fedatto et al. [15]. 
The model structure was altered to include one additional flux, corresponding to the activity of glutamate dehydrogenase, which in mammalian cells converts glutamate into alphaketogluterate, a Krebs cycle intermediate. This flux was represented in our model with simplified kinetics as follows:

$$
J_{\mathrm{gdh}}=V_{\mathrm{GDH}} \frac{[\mathrm{GLU}]_{\mathrm{x}}[\mathrm{NAD}]_{\mathrm{x}}}{K_{\mathrm{M}, \mathrm{GLU}}+[\mathrm{GLU}]_{\mathrm{x}}}
$$

The activity of glutamate dehydrogenase is a source of alphaketogluterate and NADH in the mitochondrial matrix, and consumes glutamate and $\mathrm{NAD}^{+}$. $V_{\mathrm{GDH}}$ and $K_{\mathrm{M}}$, GLU were chosen based on experimental measurements [27]. That paper uses a model of the kinetics that was even simpler, not including $\mathrm{NAD}^{+}$, for that reason, we treat that paper as measuring $V_{\max }=X_{\mathrm{GDH}} \frac{\left[N A D^{+}\right]_{x}}{K_{\mathrm{NAD}, \text { ref }}}$, where $K_{\mathrm{NAD}}$, ref is a baseline concentration of $\mathrm{NAD}^{+}$present in the mitochondrial matrix. We use physiologically reasonable concentrations to choose $K_{\mathrm{NAD}}$, ref, assume for calculation that this was the $\mathrm{NAD}^{+}$concentration present in the mitochondrial matrix in the work of Jonker et al. [27], and then $V_{\mathrm{GDH}}$ may be calculated simply as $V_{\mathrm{GDH}}=$ $\frac{X_{G D H}}{K_{\mathrm{NAD}, \text { ref }}}$. Our model requires us to model the dependence on $\mathrm{NAD}^{+}$concentration because otherwise the model may consume excessive $\mathrm{NAD}^{+}$.

\begin{tabular}{|c||c|c|}
\hline Target Quantity & Measured & Predicted \\
\hline \hline State 2 Oxygen Consumption $(\mathrm{mM} / \mathrm{s})$ & 0.30 & 0.29 \\
\hline State 3 Oligomycin-Sensitive Oxygen Consumption $(\mathrm{mM} / \mathrm{s})$ & 2.0 & 2.2 \\
\hline
\end{tabular}

Table 2: Values used to fit unknown parameters and the predicted values.

\subsection{Model Parameter Fitting}

With a minor increase (16\%) in the hydrogen leak permeability relative to the proximal tubule, we are able to find a suitable fit for the State 2 and 3 oxygen consumption as measured in isolated liver mitochondria [21]. In that work they measure the State 2 measurements of oxygen consumption to be $0.30 \mathrm{mM} / \mathrm{s}$, and the State 3 measurements of oligomycin-sensitive (or Complex IV-dependent) oxygen consumption to be $2.0 \mathrm{mM} / \mathrm{s}$. With adjustments to the hydrogen leak we get a good fit for the State 2 and 3 oxygen consumption. The results of the fit are recorded in Table 2.

\subsection{Simulations}

While there is a variety of mitochondria-mediated liver pathologies, they tend to progress through OXPHOS dysfunction or oxidative stress. The latter is attenuated by uncoupling effects, or hydrogen leak permeability. Uncoupling reduces the effect of oxidative stress because when the proton motive force is less strong, OXPHOS complexes are better able to transfer 
their electrons through the electron transport chain instead of staying in a reduced state that increases the risk of free radical formation. Oxidative stress can also initiate mitochondrial permeability transition $(\mathrm{mPT})$, which may reduce the concentration of cytochrome $\mathrm{C}$ in the intermembrane space. Oxidative stress can also produce OXPHOS dysfunction.

OXPHOS dysfunction is represented by some combination of reductions in the activities of Complex I, III, IV, and ATP Synthase $\left(X_{C I}, X_{C I I I}, X_{C I V}\right.$, and $\left.X_{F 1}\right)$. We consider combinations in reductions of these activities because many OXPHOS dysfunctions are not specific to one OXPHOS enzyme [37]. We consider successive reductions in the activity of each OXPHOS enzyme by $25 \%$ to as low as a quarter of the baseline enzyme activity.

Oxidative stress is frequently a consequence of the cell being in an energized state where the redox state of the cell's electron transport chain is too reduced. We consider two mechanisms that are able to produce oxidative stress: high glycolysis (four times the default flux) or via reperfusion following a period of ischemia (ischemia involving zero oxygen tension, smaller adenine nucleotide and nicotinamide pools). In the latter case, we consider the consequences of total hypoxia (no oxygen) over a period of 50,000 seconds (or roughly 14 hours) with a nicotinamide pool (the combined concentrations of $\mathrm{NADH}$ and $\mathrm{NAD}^{+}$) that is half the normal size, and a pool of cytosolic adenine nucleotides (the combined concentrations of ATP, ADP, and AMP) that is a quarter of normal levels [28, 58]. Following this period of ischemia, liver oxygen tension returns to normal but other features of the cell do not. We then recorded the reperfusion behaviour until it appeared to settle at a new baseline.

Aside from the above cases, we consider uncoupling on its own and hypoxia. The latter is a known consequence of liver disease, and especially alcoholic liver disease in rats $[47,55]$. We considered oxygen tensions reduced by tenths from the default oxygen tension of $36 \mathrm{mmHg}$, to a low of $3.6 \mathrm{mmHg}$. We also consider an oxygen tension of $0.5 \mathrm{mmHg}$ to gauge the effects of more extreme hypoxia. For uncoupling, we consider 2x, 5x, and 10x typical hydrogen leak permeability $\left(P_{\mathrm{H}, \text { leak }}\right)$.

\section{Results}

\subsection{Baselines}

Key model predictions are shown in Table 3. Under baseline conditions, the liver model predicts a cytosolic ATP concentration of $6.81 \mathrm{mM}$, an energy charge of 0.97 , and an ATP/ADP ratio of 14.55. These compare favourably with at least some experimental estimates. For instance Schwenke et al. measure the cytosolic ATP concentration to be $6.18 \mathrm{mM}$. The cytosolic ATP/ADP ratio was found to be 6.94 in the same study [49], although the ATP/ADP ratio varies widely between preparations $[49,62]$. Other predicted measures of the energetic state of the liver also appear to accord well with at least some experimental results. For instance the electrical potential gradient across the inner membrane of liver mitochondria was predicted to be $165 \mathrm{mV}$ (physiological conditions treated as a positive quantity). This is within experimentally observed ranges. Porter and Brand observe an electrical potential gradient of $149 \mathrm{mV}$ [46]. Other studies on isolated hepatocytes have found the electrical 


\begin{tabular}{|c|c|c|c|c|c|c|}
\hline & $\begin{array}{c}\mathrm{O}_{2} \text { Con- } \\
\text { sumption } \\
\left(\mathrm{nmol} \mathrm{O}_{2}\right. \\
\left.\mathrm{mg}^{-1} \mathrm{~s}^{-1}\right)\end{array}$ & $\begin{array}{c}\text { ATP } \\
\text { Generation } \\
(\mathrm{nmol} \\
\text { ATP } \mathrm{mg}^{-1} \\
\left.\mathrm{~s}^{-1}\right)\end{array}$ & $\mathrm{P} / \mathrm{O}$ & $\begin{array}{c}\text { Proton } \\
\text { Motive } \\
\text { Force } \\
(\mathrm{PMF}, \\
\mathrm{mV})\end{array}$ & $\begin{array}{c}{[\mathrm{ATP}]_{c}} \\
(\mathrm{mM})\end{array}$ & $\begin{array}{c}{[\mathrm{ATP}]_{c}} \\
/[\mathrm{ADP}]_{c}\end{array}$ \\
\hline \multicolumn{7}{|l|}{ Base Case } \\
\hline $\begin{array}{l}\mathrm{P}_{O 2}=36 \mathrm{mmHg} \\
\mathrm{pH}_{c}=7.20\end{array}$ & 0.59 & 2.04 & 1.72 & 168.3 & 6.81 & 14.55 \\
\hline \multicolumn{7}{|l|}{$\mathbf{P}_{O 2}$ Variation } \\
\hline $\mathrm{P}_{\mathrm{O} 2}=50 \mathrm{mmHg}$ & 0.59 & 2.04 & 1.71 & 169.49 & 6.82 & 14.88 \\
\hline $\mathrm{P}_{\mathrm{O} 2}=12 \mathrm{mmHg}$ & 0.59 & 2.04 & 1.72 & 167.67 & 6.8 & 14.30 \\
\hline $\mathrm{P}_{\mathrm{O} 2}=8 \mathrm{mmHg}$ & 0.59 & 2.04 & 1.73 & 166.56 & 6.79 & 13.87 \\
\hline \multicolumn{7}{|l|}{$\mathbf{Q}_{A T P, \max }$ Variation } \\
\hline $\mathrm{Q}_{A T P, \max } \times 0.75$ & 0.43 & 1.27 & 1.5 & 169.86 & 6.86 & 16.09 \\
\hline $\mathrm{Q}_{A T P, \max } \times 1.25$ & 0.76 & 2.8 & 1.84 & 168.32 & 6.76 & 13.19 \\
\hline $\mathrm{Q}_{A T P, \max } \times 1.5$ & 0.93 & 3.56 & 1.92 & 167.71 & 6.68 & 11.43 \\
\hline \multicolumn{7}{|l|}{$\mathbf{p H}_{c}$ Variations } \\
\hline $\mathrm{pH}_{c}=7.40$ & 0.55 & 2.03 & 1.84 & 182.01 & 6.57 & 9.68 \\
\hline $\mathrm{pH}_{c}=7.00$ & 0.65 & 2.04 & 1.57 & 155.46 & 6.97 & 21.72 \\
\hline $\mathrm{pH}_{c}=6.80$ & 0.72 & 2.01 & 1.39 & 141.79 & 7.07 & 30.95 \\
\hline \multicolumn{7}{|l|}{$\left[\mathbf{K}^{+}\right]_{c}$ Variations } \\
\hline$\left[\mathrm{K}^{+}\right]_{c}=60 \mathrm{mM}$ & 0.6 & 2.04 & 1.69 & 169.09 & 6.85 & 15.83 \\
\hline$\left[\mathrm{K}^{+}\right]_{c}=140 \mathrm{mM}$ & 0.59 & 2.03 & 1.73 & 167.82 & 6.74 & 12.62 \\
\hline \multicolumn{7}{|l|}{$\left[\mathbf{M g}^{2+}\right]_{c}$ Variations } \\
\hline$\left[\mathrm{Mg}^{2+}\right]_{c}=0.2 \mathrm{mM}$ & 0.59 & 2.03 & 1.72 & 169.01 & 6.7 & 11.56 \\
\hline$\left[\mathrm{Mg}^{2+}\right]_{c}=0.8 \mathrm{mM}$ & 0.6 & 2.05 & 1.72 & 168.98 & 6.94 & 20.01 \\
\hline \multicolumn{7}{|l|}{$\begin{array}{c}\text { Variations in } \mathbf{H}^{+} \\
\text {and } \mathbf{K}^{+} \text {leak } \\
\text { permeability }\end{array}$} \\
\hline No leaks & 0.47 & 2.09 & 2.25 & 170.38 & 6.84 & 15.47 \\
\hline $\mathrm{No} \mathrm{H}^{+}$leak & 0.52 & 2.07 & 2.0 & 169.69 & 6.82 & 14.95 \\
\hline $\begin{array}{l}\text { No } \mathrm{H}^{+} \text {leak, } \\
\text { 10x P } \mathrm{P}_{K, \text { leak }}\end{array}$ & 0.67 & 1.95 & 1.45 & 161.27 & 6.18 & 6.12 \\
\hline $\begin{array}{c}\text { No } \mathrm{K}^{+} \text {leak, } \\
10 \mathrm{x}_{H, l e a k}\end{array}$ & 1.29 & 1.76 & 0.68 & 165.15 & 6.78 & 13.51 \\
\hline $10 \mathrm{x} \mathrm{P}_{H, l e a k}$ & 1.22 & 1.78 & 0.73 & 165.11 & 6.76 & 13.21 \\
\hline 10x Leaks & 0.99 & 1.8 & 0.91 & 159.38 & 6.03 & 5.37 \\
\hline
\end{tabular}

Table 3: The model under typical conditions and various relevant deviations from normal conditions. 
potential gradient to be $154 \mathrm{mV}$ in young rats [20]. Some studies report even lower values for the electrical potential gradient such as $108 \mathrm{mV}$ [52] or $118 \mathrm{mV}$ [40], both in perfused liver. Other studies working with isolated rat liver mitochondria have reported electric potential gradients as high as $188 \mathrm{mV}$ [45]. In the above cases, the proton motive force was also frequently measured. There is a wide range of experimental measurements, which encompasses our predictions, we predicted the proton motive force to be $168 \mathrm{mV}$. Experiments have found the proton motive force to range from $125 \mathrm{mV}$ [52] to $191 \mathrm{mV}$ [20].

We calculate the $\mathrm{P} / \mathrm{O}$ ratio only taking into account oxygen consumption by the mitochondria. Unlike multiple experimental studies, the $\mathrm{P} / \mathrm{O}$ ratio is calculated using only mitochondrial oxygen consumption (as well with non-mitochondrial oxygen consumption) by Brand, Harper, and Taylor. Including only mitochondrial oxygen consumption, they find a $\mathrm{P} / \mathrm{O}$ ratio of 1.69 [7]. This is comparable to our predicted $\mathrm{P} / \mathrm{O}$ ratio of 1.72 . The $\mathrm{P} / \mathrm{O}$ ratio was calculated from the ATP generation, which under default conditions was found to be $2.04 \mathrm{nmol}$ ATP $\mathrm{mg}^{-1}$ prot $\mathrm{s}^{-1}$ by ATP Synthase, and $\mathrm{O}_{2}$ consumption which was found to be $0.59 \mathrm{nmol} \mathrm{O}_{2} \mathrm{mg}^{-1}$ prot $\mathrm{s}^{-1}$ (the $\mathrm{P} / \mathrm{O}$ ratio is the ATP generation (or ATP Synthase activity) divided by $2 \mathrm{x}$ the oxygen consumption (or Complex IV activity)).

\subsection{Parameter Sensitivity Analysis}

Here we vary several important parameters, namely the leak permeabilities, cytosolic ion concentrations, maximal ATP consumption, and oxygen tension. For this analysis we consider large changes to these parameters and report the effects (the exact changes are noted in the first column of Table 3), rather than reporting local measures of sensitivity (see Table 3). Our sensitivity analysis indicates that only some of these perturbations have a strong impact on liver mitochondrial function, notably maximal ATP consumption and leak permeabilities.

The key parameters that most significantly alter the system's behaviour in the ranges considered here are the maximal ATP consumption and the leak permeabilities $\left(P_{\mathrm{H} \text {, leak }}\right.$ and $\left.P_{\mathrm{K} \text {, leak }}\right)$. Under a 1.5-fold increase in maximal ATP consumption, there's a slightly more than 1.6-fold increase in oxygen consumption. This suggests a larger maximal ATP consumption could lead to hypoxia, due to the significantly increased oxygen consumption. However the efficiency as measured by the $\mathrm{P} / \mathrm{O}$ ratio of respiration also improves with increase ATP consumption (for higher ATP consumption, the $\mathrm{P} / \mathrm{O}$ ratio may be as high as 1.92). Leak permeabilities also had a major impact on the oxygen consumption. When there is no potassium leak and a ten-fold increase in hydrogen leak, the oxygen consumption more than doubles. When there is a ten-fold increase in potassium and hydrogen leak, the ATP concentration reaches its minimum, $6.03 \mathrm{mM}$, the ATP/ADP ratio hits its minimum as well of 5.37, as did the proton motive force, which gets to a minimum of $159 \mathrm{mV}$.

These results suggest that uncoupling and greater ATP demand could trigger greater oxygen consumption, possibly producing hypoxic conditions. 
bioRxiv preprint doi: https://doi.org/10.1101/2021.12.17.473135; this version posted December 19, 2021. The copyright holder for this preprint (which was not certified by peer review) is the author/funder, who has granted bioRxiv a license to display the preprint in perpetuity. It is made available under aCC-BY-ND 4.0 International license.

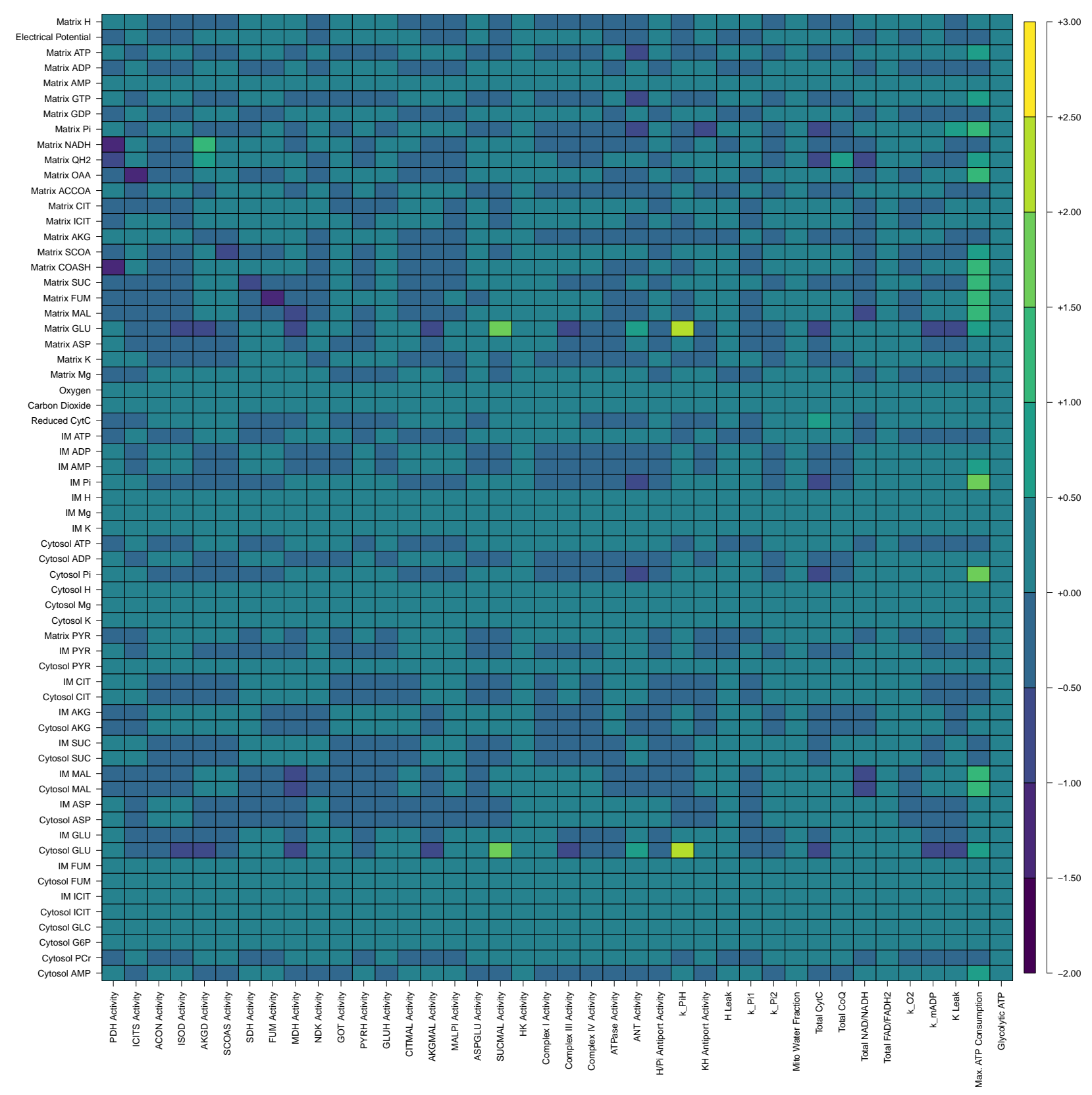

Figure 1: The full set of local sensitivities in the liver, for more information see section 3.3. CytC stands for cytochrome $\mathrm{C}$, reduced cytochrome $\mathrm{C}$ refers to cytochrome $\mathrm{C}$ that has been donated an electron by Complex III.

\subsection{Local Sensitivity Analysis}

We consider the change in state variables in hepatocytes under a change in each parameter of our model. Below we show the derivative of each state variable against each parameter, 
calculated using a central difference scheme with $\Delta p=0.01 p$ where $p$ is the size of the parameter. The derivative is normalized relative to the baseline size of each state variable and the size of each parameter. The full local sensitivity analysis is reported in Figure 1. In Figure 2 we focus on several key variables and the parameters that are most impactful on those state variables. Hepatocytes in the liver appear to be fairly sensitive to many parameters. Several sensitivities are much higher than the comparable sensitivities in the PT and mTAL respectively. The NADH and reduced coenzyme Q concentrations were particularly sensitive to multiple key parameters. Cytosolic ATP concentrations are most sensitive to the maximal ATP consumption. The mechanism explaining the response of NADH concentrations to the activities of pyruvate dehydrogenase and alphaketogluterate dehydrogenase is the same as in the proximal tubule discussed above. Reduced coenzyme Q concentrations are strongly reactive to total $\mathrm{NADH} / \mathrm{NAD}^{+}$concentrations, and thus to pyruvate dehydrogenase and alphaketogluterate dehydrogenase activity. Aside from those effects, the reduced coenzyme Q concentration is strongly sensitive to the total available coenzyme Q, and the pool available of cytochrome $\mathrm{C}$, which is negatively associated with the quantity of reduced coenzyme $\mathrm{Q}$. This indicates that the concentration of reduced coenzyme $\mathrm{Q}$ is restricted by the total amount of coenzyme $\mathrm{Q}$ to a moderate extent, and that the reduction state of the coenzyme $\mathrm{Q}$ is also controlled by the available cytochrome $\mathrm{C}$ to accept electrons from coenzyme Q. Overall this indicates that the coenzyme $\mathrm{Q}$ and cytochrome $\mathrm{C}$ pools are not present in great excess of what the mitochondrion needs.

\subsection{OXPHOS Dysfunction}

We describe our method for simulating dysfunction of oxidative phosphorylation (OXPHOS), that is activity of electron transport chain components and ATP synthase, above in Section 2.3. With a lowest OXPHOS complex activity of one quarter, there is little discernable consequence for cytosolic ATP consumption, and very little noticeable difference in the electrical potential gradient. With reduction to one quarter of all considered OXPHOS complex activities, the cytosolic ATP concentration differed by less than $2 \%$. The electrical potential gradient never differed by more than 4\%. Thus, impaired OXPHOS function was found to have little impact on ATP concentrations, or the electrical potential gradient of the liver. This result is consistent with the relatively few mitochondrial diseases known to affect the liver [37]. Those that do tend to be particularly severe, causing death in childhood, or progressive diseases leading ultimately to the depletion of mitochondrial DNA. The results were not significantly different under moderate hypoxia or reduced glycolysis.

Liver hepatocytes are predicted to be more robust than the proximal tubule (PT) of the kidney to OXPHOS dysfunction. The thick ascending limb (mTAL) is also more sensitive to OXPHOS dysfunction than the liver, but it has more confounding differences in tissue oxygen tension and mitochondrial volume fraction. We explore the reason for the difference between the PT and liver in robustness by considering the effect of reducing Complex III activity by $75 \%$ for hepatocyte mitochondria with PT-like parameter values for metabolic demand, glycolysis activity, or baseline OXPHOS enzyme activities (and combinations thereof). We 


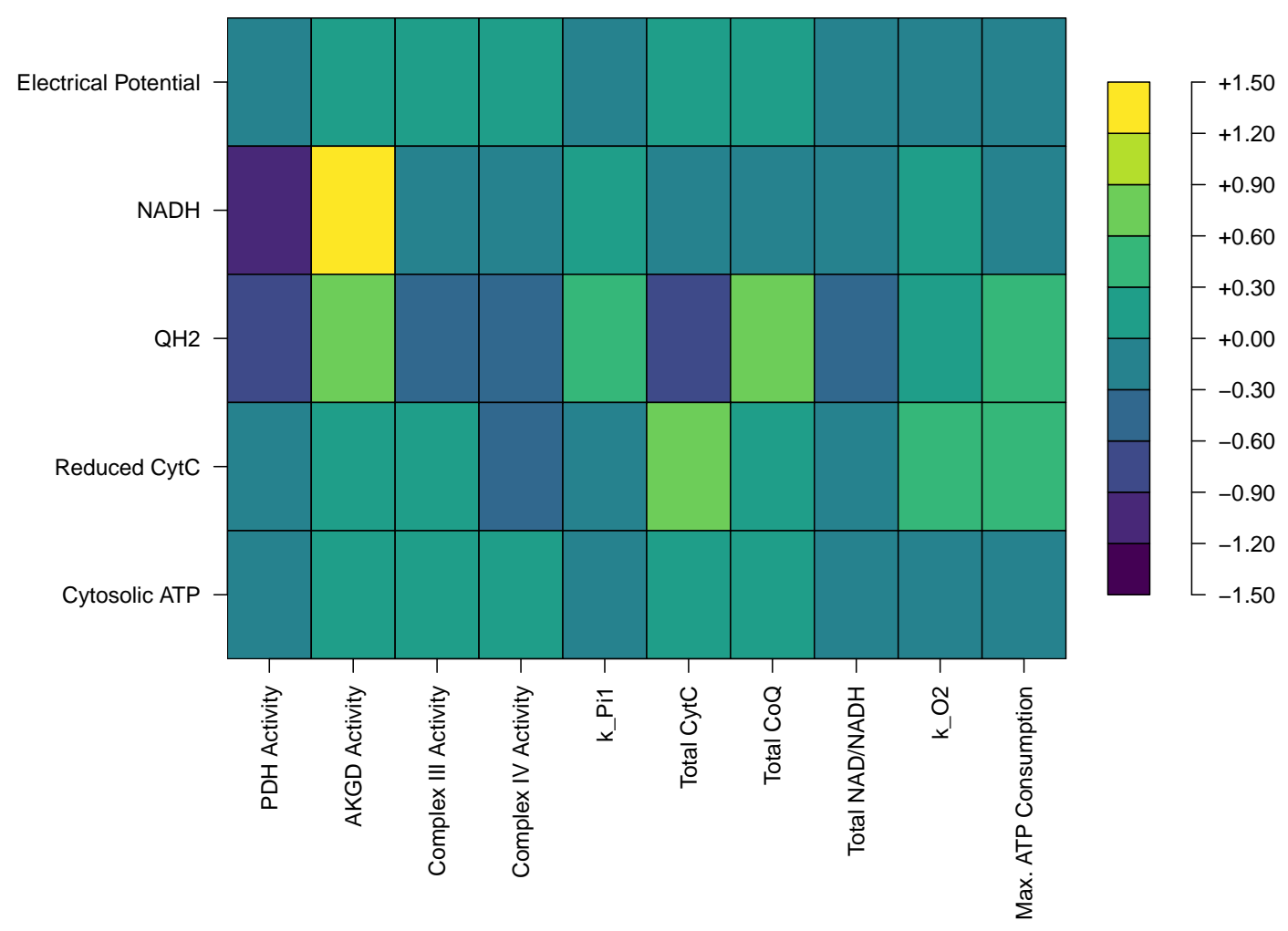

Figure 2: Local sensitivities of several important state variables in hepatocytes relative to certain significant parameters. CytC stands for cytochrome $\mathrm{C}$, reduced cytochrome $\mathrm{C}$ refers to cytochrome $\mathrm{C}$ that has been donated an electron by Complex III. AKGD refers to alphaketogluterate dehydrogenase, $\mathrm{PDH}$ refers to pyruvate dehydrogenase, ANT refers to adenine nucleotide translocase, and $\mathrm{QH}_{2}$ is the reduced form of coenzyme $\mathrm{Q}$.

consider another kind of robustness that the liver hepatocytes exhibit below (namely, to hypoxia). The PT has $76 \%$ of baseline cytosolic ATP concentrations under reduced Complex III activity (a difference of $0.6 \mathrm{mM}$ ). With no glycolysis (matching the PT) or PT-like baseline OXPHOS activities, the difference in cytosolic ATP concentration is negligible for a liver hepatocyte with Complex III dysfunction. With PT-like metabolic demand, we see $94 \%$ of baseline cytosolic ATP concentrations in hepatocytes (a difference of $0.4 \mathrm{mM}$ ). With both no glycolysis and PT-like metabolic demand, we see $87 \%$ of baseline cytosolic ATP concentrations (a difference of $0.9 \mathrm{mM}$ ). With PT-like OXPHOS activities and metabolic demand, we see $86 \%$ of baseline cytosolic ATP concentrations during Complex III dysfunction. With no glycolysis and PT-like baseline OXPHOS activities, we see negligible differences from baseline cytosolic ATP concentrations. With no glycolysis, PT-like metabolic demand, and PT-like baseline OXPHOS activities, we see $71 \%$ of baseline cytosolic ATP concentrations (a difference of $2 \mathrm{mM}$ ). This indicates that adjusting the glycolytic activity, OXPHOS activities, and metabolic demand to PT-like parameters is necessary and together sufficient for us to 


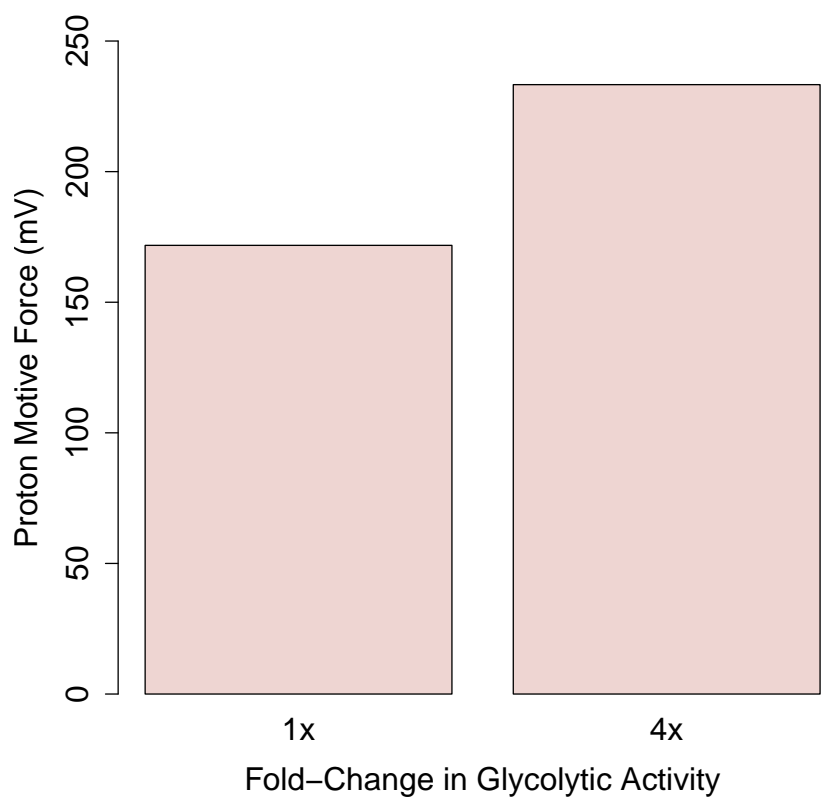

Figure 3: The left is the steady state proton motive force for the baseline model, and the right is the value under high rates of glycolysis (a quadrupled glycolytic activity).

observe PT-like sensitivity to OXPHOS dysfunction.

\subsection{Conditions producing Oxidative Stress}

Oxidative stress can occur under a range of circumstances. In our simulation work, high rates of glycolysis appear to be a particularly significant potential means of producing oxidative stress. We see in Figure 3 that under these glycolytic conditions, the proton motive force is extremely high, supporting this conclusion. We consider high rates of maximal glcolysis, four times our default, similar to those observed in the presence of $20 \mathrm{mM}$ of glucose [15].

\subsection{Hypoxia}

Our hypoxic simulations are outlined above in Section 2.3. The liver is only sensitive to ATP depletion under sufficiently large reductions in the oxygen tension in mitochondria. At an oxygen tension of $0.5 \mathrm{mmHg}$ the cytosolic ATP concentration is reduced by $15 \%$ (to $4.3 \mathrm{mM}$ ). However at a tenth of normoxic oxygen tension $(3.6 \mathrm{mmHg})$, cytosolic ATP concentrations are reduced by less than $2 \%$. These results may seem inconsistent with the understood possibility of hypoxic liver injury but they are not. In both ischemia and alcohol exposure in chronic alcoholic liver disease, we see that oxygen tensions can locally get as low as can be measured quite frequently $[39,47]$. Thus, it is possible that under certain kinds of disruptions of normal oxygen tension, the mitochondrial matrix becomes extremely hypoxic (below $1 \mathrm{mmHg}$ oxygen 
tension). Mechanistically the robustness of the model to hypoxia can be explained in part by the relatively high rates of (anaerobic) glycolysis observed in liver tissue [15], this allows the tissue to continue fulfilling its ATP needs under mitochondrial dysfunction.

Comparing ATP depletion between liver hepatocytes and the kidney, we see that hepatocytes are more robust to hypoxia. We simulate hepatocytes with PT-like metabolic demand, glycolysis, and baseline OXPHOS activity in order to determine the differences that explain why they differ. We choose to compare to the PT because of the more comparable baseline oxygen tension. With $10 \%$ of baseline oxygen tension, the PT has $19 \%$ of its typical cytosolic ATP concentration (a difference of $2.1 \mathrm{mM}$ ). For PT-like metabolic demand, we have $73 \%$ of typical cytosolic ATP concentrations in the hypoxic hepatocyte (a difference of $1.9 \mathrm{mM}$ ). Without glycolysis, we have $96 \%$ of typical cytosolic ATP concentrations in the hypoxic hepatocyte (a difference of $0.3 \mathrm{mM}$ ). With both higher metabolic demand and no glycolysis, we have $61 \%$ of typical cytosolic ATP concentrations in the hypoxic hepatocytes (a difference of $2.7 \mathrm{mM}$ ). With PT-like metabolic demand, no glycolysis, and PT-like OXPHOS activity, we see $39 \%$ of typical cytosolic ATP concentrations (a difference of $4.2 \mathrm{mM}$ ).

\subsection{Uncoupling}

Uncoupling is used by the cell as a means of relieving oxidative stress. However uncoupling also directly reduces the $\mathrm{P} / \mathrm{O}$ ratio governing the efficiency of ATP generation, and increases oxygen consumption [48, 59], which may cause hypoxia. Titrating with FCCP, a potent uncoupler, until the respiratory rate was maximal, Schönfeld et al. was able to experimentally produce a $60 \%$ increase in oxygen consumption [48]. Our model reproduces this proportional respiration rate increase with a five-fold increase in hydrogen leak. Under a two fold increase in the hydrogen leak activity, we predict a $17 \%$ increase in oxygen consumption, which in an organ with already quite significant oxygen gradients might be quite important. Already under a two-fold increase in the hydrogen leak, the $\mathrm{P} / \mathrm{O}$ ratio predicted by the model to be as low as 1.32 , compared to a baseline of 1.72 , a $24 \%$ decrease.

\subsection{Ischemia-Reperfusion Injury}

Ischemia-reperfusion causes oxidative stress by leaving the electron transport chain in a highly reduced state temporarily. This is dangerous to the cell and the body, and can even lead to multiple organ dysfunction [43]. We wish to capture ischemia-reperfusion injury in our model and consider some candidate methods for preventing ischemia-reperfusion injury. To achieve this, we will simulate ischemia, and then we will simulate reperfusion in multiple ways. Then, we will study reperfusion in combination with interventions using pyruvate and dichloroacetate. During ischemia, the cell experiences a decline in both pyruvate and oxygen concentrations. As a consequence of ischemia, the adenine nucleotide (AMP/ADP/ATP) and nicotinamide adenine dinucleotide $\left(\mathrm{NADH} / \mathrm{NAD}^{+}\right)$pools become significantly smaller. The former shrinks by three quarters $[28,58]$, and the latter by one half [28]. Ischemia also causes OXPHOS dysfunction that reduces the activity of Complex I, III, and IV. 

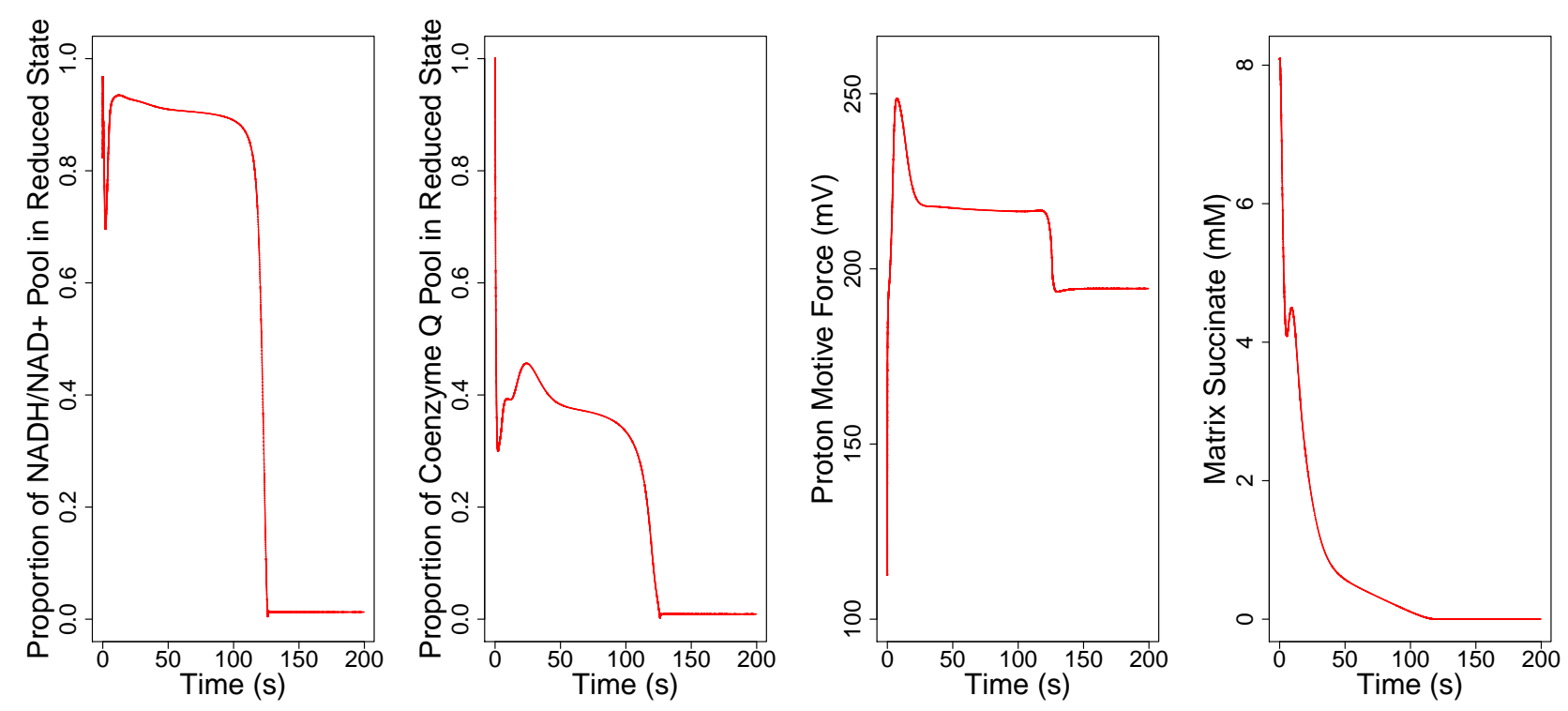

Figure 4: The effects of reperfusion with a smaller pool of adenine nucleotides on $\mathrm{NADH} / \mathrm{NAD}^{+}$\& coenzyme $\mathrm{Q}$ redox state, the proton motive force, and the succinate concentration.

For our model, we consider a shrunken pool of AMP, ADP, ATP, NADH, and NAD ${ }^{+}$ during reperfusion on top of restoration of typical oxygen levels after a bout of severe hypoxia (0 mmHg). We shrink the pool of adenine nucleotides by three quarters and the pool of nicotinamide adenine nucleotides by half following experimental results from Kamiike et al [28]. In this case, shown in Figure 4, we see that the $\mathrm{NADH} / \mathrm{NAD}^{+}$and coenzyme $\mathrm{Q}$ pools are in a significantly reduced state for several minutes after reperfusion, and that the proton motive force is extremely elevated during the same period of time. This matches the timescale expected from experimental work, where it has been observed that reactive oxygen species are produced rapidly in a short period following reperfusion or reoxygenation [26]. In another trial, we consider the consequences of also including OXPHOS dysfunction. We include OXPHOS dysfunction by reducing the activity of Complex I, III, and IV by 15\%, 70\%, and $50 \%$ respectively following experimental results from Baniene et al. [63]. Under these circumstances, shown in Figure 5, we predictably see a somewhat more reduced coenzyme Q pool with a somewhat lower but still very high proton motive force. This is to be expected because a reduced rate of oxidative phosphorylation means that it will take longer to oxidize coenzyme Q. In other trials, we consider more moderate hypoxia (one tenth of typical) and we do not observe the same long transients. This is demonstrably due to a lack of succinate build up because when we increase the succinate levels in these trials to levels comparable to our more extreme hypoxic case, we again see long transients. 

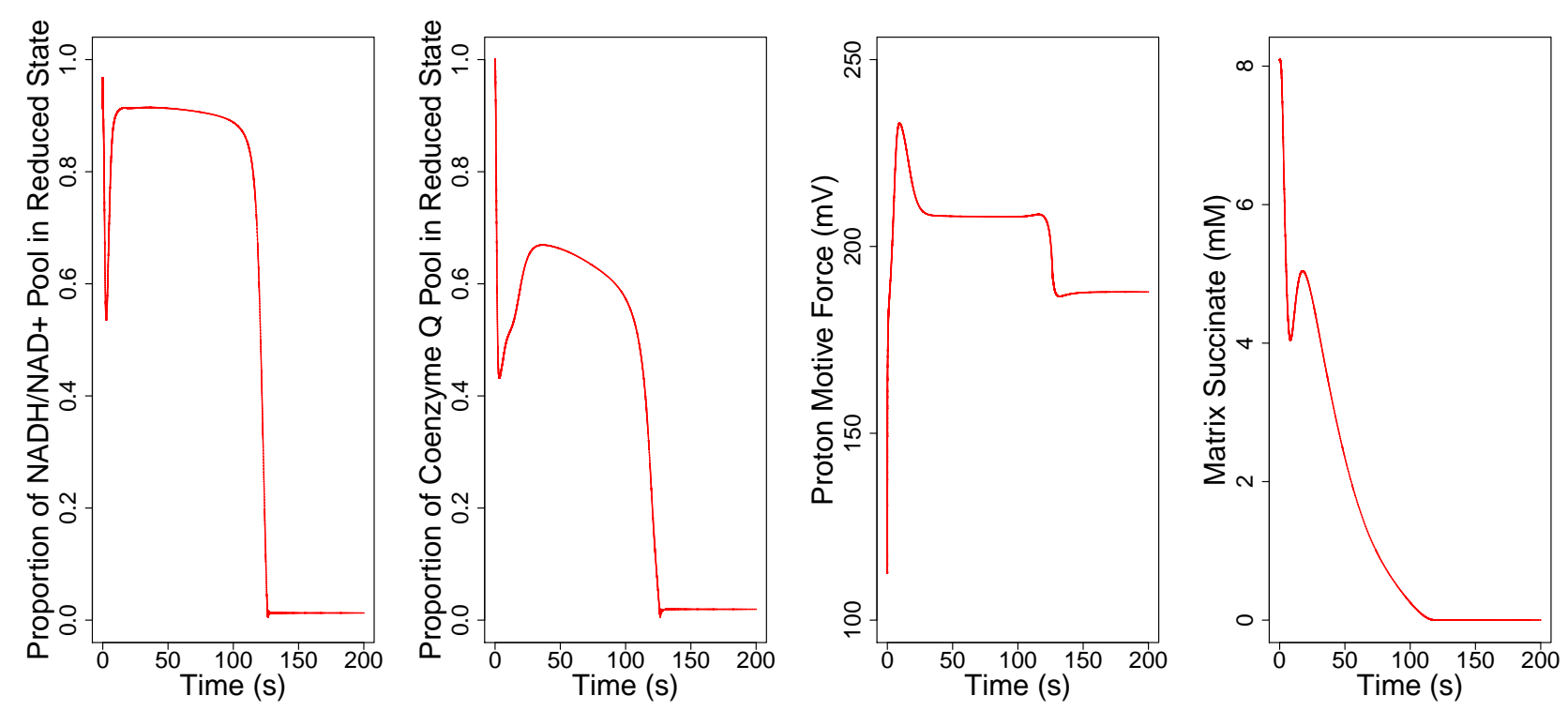

Figure 5: The effects of reperfusion with a smaller pool of adenine nucleotides and OXPHOS dysfunction on $\mathrm{NADH} / \mathrm{NAD}^{+}$redox state, coenzyme Q redox state, and proton motive force.

\section{Discussion}

Our model simulations tell a simple story: the liver is highly robust to ATP depletion, but possibly quite sensitive to phenomena known to cause oxidative stress. These two features of liver mitochondria happen for overlapping reasons. The liver has a high capacity for glycolytic activity [15]. This capacity means the mitochondria do not have to satisfy the hepatocyte's metabolic burden on their own, but also can leave the mitochondria in a highly reduced state for lack of ADP to phosphorylate. This underlies our findings shown in Figure 3 that at the rates of glycolysis observed by Fedatto et al. [15] in healthy rats experiencing hyperglycemia, the electrical potential gradient is larger across the inner membrane of the mitochondria. This trade off of, on the one hand, being able to satisfy the cell's energetic requirements but on the other, risking oxidative stress, appears to be valuable for interpreting our results. We note reasons for this robustness in Sections 3.4 and 3.6. Our results demonstrate that it is a combination of several factors that explains the differences in robustness observed in liver hepatocytes compared to the proximal tubule in the kidney, a tissue that is far less robust to ATP depletion. Our results indicate that the liver's glycolytic activity, lower metabolic demand, and higher basline OXPHOS activites all contribute to the liver's robustness to dysfunction of Complex III, the rate-limiting step of the electron transport chain, and all must be changed to PT-like values in order to produce PT-like sensitivity to OXPHOS dysfunction. Similarly we need to change all three of these features of the hepatocyte in order to see PT-like sensitivity to ATP depletion during hypoxia. The hepatocyte simply has more electron transport activity servicing a smaller metabolic demand with a larger contribution from glycolysis, and this makes it more robust to significant kinds of ATP depletion. 
This ability to satisfy the cell's energetic requirements may be examined in several ways. First of all, the cell is predicted to maintain a steady concentration of ATP even under a $50 \%$ increase in the maximal ATP consumption, as shown in Table 3. Second, as noted above in Section 3.4, if the rates of glycolysis are reduced we also see that the mitochondria can make up the difference by increasing their ATP generation. Each of these model simulations support our contention that the mitochondria may robustly provide for the hepatocyte's ATP needs. These observations should not surprise us, since ATP depletion is not frequently observed in mitochondria-mediated liver pathologies, unlike oxidative stress [61, 41].

Ischemia-reperfusion injury is frequently caused by a temporary excessively reduced redox state of the electron transport chain causing oxidative stress during reperfusion [6]. We represent ischemia in our model by decreased oxygen tension and pyruvate concentration. During reperfusion we include a decreased supply of adenine nucleotides, based on a study that indicates a $75 \%$ smaller adenine nucleotide following ischemia [28]. We observe a transient redox state change as recorded in Figure 4. We also consider added effects from OXPHOS dysfunction. When we include these effects, we see a more reduced coenzyme Q pool and a marginally lower transient proton motive force relative to the ischemia-reperfusion case without OXPHOS dysfunction, as shown in Figure 5. Both resemble experimentally observed timescales for the enhanced production of $\operatorname{ROS}$ following ischemia $[11,19,26]$. However the results in the latter case should be interpreted carefully. Reperfusion injury harms the electron transport chain, and it is often difficult to study the effects of ischemia on oxidative phosphorylation independently from the effects of reperfusion. For this reason, the levels of OXPHOS dysfunction used in Figure 5 should be treated as potential overestimates. These limitations are not a major problem because we are able to reproduce the appropriate timescale of reperfusion transients with or without OXPHOS dysfunction as noted above.

The saturation of the electron transport chain with electrons during reperfusion appears to be closely tied to the build up of succinate in ischemia, and the associated consumption of succinate following reperfusion. This coincides with suggested mechanisms for reperfusion injury in cardiac tissue [11]. As Chouchani et al. [11] notes, succinate build up has a preponderance of evidence indicating its importance to mitochondria-mediated ischemiareperfusion injury. Under more moderate hypoxia in other cases considered, where succinate build up is not observed in the same way, we do not see the same indicators of risk of ROS production that we note above.

In rats, it has been observed that chronic alcohol consumers have worse hepatic oxygen homeostasis relative to control non-alcoholic rats [47]. That is, in the event of acute alcohol consumption, chronic alcohol consumers may experience extremely low hepatic oxygen tensions. Sato et al. found that chronic alcohol consumers had a median oxygen tension upon acute alcohol consumption of $8 \mathrm{mmHg}$ [47]. This is comparable to the hypoxic simulation case discussed in the previous paragraph. This suggests a mechanism by which alcohol consumption in individuals with alcoholic liver disease may trigger oxidative stress in a tissue, accelerating the progression of the disease.

While glycolysis may be elevated to levels like those noted previously in severe hyperglycemia, there are other ways to produce comparably elevated glycolysis as well. Fedatto 
et al. [15] studied hepatic tissue of rats with adjuvant arthritis (triggered by heat-killed Mycobacterium tuberculosis). The maximal rate of glycolysis was found to be roughly $30 \%$ higher in the arthritic rats. This presents another means of producing elevated glycolysis, which is potentially a trigger for oxidative stress. Fedatto et al. hypothesize other illnesses causing circulating inflammatory mediators may produce the same glycolytic effects [15], suggesting a range of diseases that could be causing oxidative stress in hepatocytes.

Our work can predict various indicators of oxidative stress, but cannot predict ROS production or the subsequent oxidative stress. Future work could our model with realistic models of ROS production. The former problem may be resolved by adapting existing models of ROS production [17] to the liver and incorporating them into our model. Predicting oxidative stress requires progress in the study of mitohormesis for the liver, so that thresholds for pathological ROS production may be found. As it stands we know that a moderate amount of ROS production may be a good thing [44]. Another contribution that could improve our model would be to integrate it with models of other pathways of hepatic metabolism, models of this kind exist for at least parts of hepatic metabolism [12]. Finally, our parameter estimates draw from a heterogeneous set of sources, including some experimental estimates from mice, namely, the Na-K-ATPase activity (discussed in Section 2.1), the cytosolic potassium concentration [57], and the pooled $\mathrm{NADH} / \mathrm{NAD}^{+}$concentration [8]. With more data from rats, it is possible we would have better predictions.

We include glutamate dehydrogenase activity in our model. Jonker et al. [27] found sexual dimorphism in the parameter $K_{\mathrm{NAD}}$, ref in rat hepatocytes. For the purpose of this work an intermediate value was chosen between the sexes, but future work could expand on ours by creating a sex-specific version of the model that included this sexual dimorphism in glutamate dehydrogenase dynamics. Valle et al. [56] notes sexual dimorphism in OXPHOS capacity and mitochondrial protein content as well. These differences are likely to be the most impactful on mitochondrial behaviour since as we mentioned before, OXPHOS capacity is a key factor that explains the liver's robustness to OXPHOS dysfunction.

Our results, obtained in this study and published studies, indicate that different tissues have characteristic ways that they are sensitive to mitochondrial dysfunction, or mitochondriamediated injury. These ways can be connected to a range of mitochondrial and tissue-scale factors. For instance, the activity of OXPHOS enzymes is crucial to the robustness of the liver and is a mitochondrial-scale factor. On the other hand, tissue-scale factors such as high metabolic demand (necessary in the kidney for instance for urine concentration [1]) and oxygen tension appear in our model to be crucial for understanding the risks from mitochondrial diseases. This opens up space for extending our model: we know that the tissue may affect the mitochondria, but in aggregate, mitochondria may also affect the tissue. A limitation of our model is that tissue-scale factors are not modelled as dynamically responsive to mitochondrial functioning. For example, oxygen tension is clamped in our model, meaning that regardless of local oxygen consumption the tissue's oxygen tension remains the same. Consequently, if we want to understand the effect of a certain degree of uncoupling (which increases oxygen consumption), we have no better method of pursuing this than simply seeing how it affects the mitochondria to combine that level of uncoupling with various degrees of hypoxia, 
without any way of predicting the appropriate degree of hypoxia. This limitation points towards a corresponding ambition however: multi-scale modelling of metabolism. We believe the best way to expand on this work is to integrate it into larger models of cellular transport $[23,31,24,22,34,36,33,35,32,38,13,30]$ and oxygenation $[51,50,9,10,16]$ and of the factors determining local metabolic demand. Appropriate multi-scale models of metabolism will involve more than just placing our mitochondrial models inside of a larger scale (and ultimately, hopefully whole-body) model with appropriate interfaces. While the liver and kidney are particularly important to whole-body homeostasis, every tissue has mitochondria and most tissues are susceptible to mitochondrial dysfunction or mitochondria-mediated injury. For a whole-body multiscale model of metabolism then, we need models of mitochondrial respiration in other tissues.

In conclusion, we have developed a model of hepatocytes in mitochondria that predicts ATP generation, oxygen consumption, and risk factors of ROS production \& oxidative stress. Our findings agree with empirical findings that ATP depletion is not a major risk in the liver, and seems to support the view that oxidative stress might be important to liver pathophysiology in many cases.

\section{References}

[1] Aw Mun, Armstrong Tamara M, Nawata C Michele, Bodine Sarah N, Oh Jeeeun J, Wei Guojun, Evans Kristen K, Shahidullah Mohammad, Rieg Timo, and Pannabecker Thomas L. Body mass-specific na+-k+-atpase activity in the medullary thick ascending limb: implications for species-dependent urine concentrating mechanisms. American Journal of Physiology-Regulatory, Integrative and Comparative Physiology 314(4): R563R573, 2018.

[2] Aw TY and Jones Dean P. Atp concentration gradients in cytosol of liver cells during hypoxia. American Journal of Physiology-Cell Physiology 249(5): C385-C392, 1985.

[3] Beauvoit B, Kitai T, and Chance B. Contribution of the mitochondrial compartment to the optical properties of the rat liver: a theoretical and practical approach. Biophysical Journal 67(6): 2501-2510, 1994.

[4] Ben-Moshe Shani and Itzkovitz Shalev. Spatial heterogeneity in the mammalian liver. Nature Reviews Gastroenterology \& Hepatology 16(7): 395-410, 2019.

[5] Benard Giovanni, Faustin Benjamin, Passerieux Emilie, Galinier Anne, Rocher Christophe, Bellance Nadege, Delage J-P, Casteilla Louis, Letellier Thierry, and Rossignol Rodrigue. Physiological diversity of mitochondrial oxidative phosphorylation. American Journal of Physiology-Cell Physiology 291(6): C1172-C1182, 2006.

[6] Bi Jianbin, Zhang Jia, Ren Yifan, Du Zhaoqing, Li Qingshan, Wang Yue, Wei Shasha, Yang Lifei, Zhang Jingyao, Liu Chang, and others . Irisin alleviates liver ischemia- 
reperfusion injury by inhibiting excessive mitochondrial fission, promoting mitochondrial biogenesis and decreasing oxidative stress. Redox biology 20: 296-306, 2019.

[7] Brand Martin D, Harper Mary-Ellen, and Taylor Heather C. Control of the effective p/o ratio of oxidative phosphorylation in liver mitochondria and hepatocytes. Biochemical Journal 291(3): 739-748, 1993.

[8] Camacho-Pereira Juliana, Tarragó Mariana G, Chini Claudia CS, Nin Veronica, Escande Carlos, Warner Gina M, Puranik Amrutesh S, Schoon Renee A, Reid Joel M, Galina Antonio, and others . Cd38 dictates age-related nad decline and mitochondrial dysfunction through an sirt3-dependent mechanism. Cell metabolism 23(6): 1127-1139, 2016.

[9] Chen Jing, Edwards Aurélie, and Layton Anita T. Effects of ph and medullary blood flow on oxygen transport and sodium reabsorption in the rat outer medulla. American Journal of Physiology-Renal Physiology 298(6): F1369-F1383, 2010.

[10] Chen Jing, Sgouralis Ioannis, Moore Leon C, Layton Harold E, and Layton Anita T. A mathematical model of the myogenic response to systolic pressure in the afferent arteriole. American Journal of Physiology-Renal Physiology 300(3): F669-F681, 2011.

[11] Chouchani Edward T, Pell Victoria R, Gaude Edoardo, Aksentijević Dunja, Sundier Stephanie Y, Robb Ellen L, Logan Angela, Nadtochiy Sergiy M, Ord Emily NJ, Smith Anthony C, and others. Ischaemic accumulation of succinate controls reperfusion injury through mitochondrial ros. Nature 515(7527): 431-435, 2014.

[12] Cvitanović Tomaš Tanja, Urlep Žiga, Moškon Miha, Mraz Miha, and Rozman Damjana. Liversex computational model: Sexual aspects in hepatic metabolism and abnormalities. Frontiers in physiology 9: 360, 2018.

[13] Edwards Aurélie, Castrop Hayo, Laghmani Kamel, Vallon Volker, and Layton Anita T. Effects of nkcc2 isoform regulation on nacl transport in thick ascending limb and macula densa: a modeling study. American Journal of Physiology-Renal Physiology 307(2): F137-F146, 2014.

[14] Edwards Aurélie, Palm Fredrik, and Layton Anita T. A model of mitochondrial o2 consumption and atp generation in rat proximal tubule cells. American Journal of Physiology-Renal Physiology 318(1): F248-F259, 2020.

[15] Fedatto Z Jr, Ishii-Iwamoto EL, Bersani-Amado C, Maciel ER Martins, Bracht A, and Kelmer-Bracht AM. Glucose phosphorylation capacity and glycolysis in the liver of arthritic rats. Inflammation Research 49(3): 128-132, 2000.

[16] Fry Brendan C, Edwards Aurélie, Sgouralis Ioannis, and Layton Anita T. Impact of renal medullary three-dimensional architecture on oxygen transport. American Journal of Physiology-Renal Physiology 307(3): F263-F272, 2014. 
[17] Gauthier Laura D, Greenstein Joseph L, Cortassa Sonia, O’Rourke Brian, and Winslow Raimond L. A computational model of reactive oxygen species and redox balance in cardiac mitochondria. Biophysical journal 105(4): 1045-1056, 2013.

[18] Geerts Albert. 2001, p. 311-336.

[19] Granger D Neil and Kvietys Peter R. Reperfusion injury and reactive oxygen species: the evolution of a concept. Redox biology 6: 524-551, 2015.

[20] Hagen Tory M, Yowe David L, Bartholomew James C, Wehr Carol M, Do Katherine L, Park Jin-Y, and Ames Bruce N. Mitochondrial decay in hepatocytes from old rats: membrane potential declines, heterogeneity and oxidants increase. Proceedings of the National Academy of Sciences 94(7): 3064-3069, 1997.

[21] Hall Arnaldur, Larsen Anna K, Parhamifar Ladan, Meyle Kathrine D, Wu Lin-Ping, and Moghimi S Moein. High resolution respirometry analysis of polyethylenimine-mediated mitochondrial energy crisis and cellular stress: Mitochondrial proton leak and inhibition of the electron transport system. Biochimica et Biophysica Acta (BBA)-Bioenergetics 1827(10): 1213-1225, 2013.

[22] Hu Rui and Layton Anita. A computational model of kidney function in a patient with diabetes. International Journal of Molecular Sciences 22(11): 5819, 2021.

[23] Hu Rui, McDonough Alicia A, and Layton Anita T. Functional implications of the sex differences in transporter abundance along the rat nephron: modeling and analysis. American Journal of Physiology-Renal Physiology 317(6): F1462-F1474, 2019.

[24] Hu Rui, McDonough Alicia A, and Layton Anita T. Sex differences in solute transport along the nephrons: effects of na+ transport inhibition. American Journal of PhysiologyRenal Physiology 319(3): F487-F505, 2020.

[25] Iannello Silvia, Milazzo Paolina, and Belfiore Francesco. Animal and human tissue na, k-atpase in obesity and diabetes: a new proposed enzyme regulation. The American journal of the medical sciences 333(1): 1-9, 2007.

[26] Jaeschke Hartmut and Mitchell Jerry R. Mitochondria and xanthine oxidase both generate reactive oxygen species in isolated perfused rat liver after hypoxic injury. Biochemical and biophysical research communications 160(1): 140-147, 1989.

[27] Jonker Ard, Geerts Willie JC, Charles Rob, Lamers Wouter H, and Van Noorden Cornelis JF. The dynamics of local kinetic parameters of glutamate dehydrogenase in rat liver. Histochemistry and cell biology 106(4): 437-443, 1996.

[28] Kamiike Wataru, Watanabe Fusao, Hashimoto Tadao, Tagawa Kunio, Ikeda Yoshikazu, Nakao Kazuyasu, and Kawashima Yasunaru. Changes in cellular levels of atp and its catabolites in ischemic rat liver. The Journal of Biochemistry 91(4): 1349-1356, 1982. 
[29] Lambert AJ, Wang B, Yardley J, Edwards J, and Merry BJ. The effect of aging and caloric restriction on mitochondrial protein density and oxygen consumption. Experimental gerontology 39(3): 289-295, 2004.

[30] Layton Anita T, Edwards Aurélie, and Vallon Volker. Adaptive changes in gfr, tubular morphology, and transport in subtotal nephrectomized kidneys: modeling and analysis. American Journal of Physiology-Renal Physiology 313(2): F199-F209, 2017.

[31] Layton Anita T, Edwards Aurélie, and Vallon Volker. Renal potassium handling in rats with subtotal nephrectomy: modeling and analysis. American Journal of PhysiologyRenal Physiology 314(4): F643-F657, 2018.

[32] Layton Anita T, Laghmani Kamel, Vallon Volker, and Edwards Aurélie. Solute transport and oxygen consumption along the nephrons: effects of na+ transport inhibitors. American Journal of Physiology-Renal Physiology 311(6): F1217-F1229, 2016.

[33] Layton Anita T and Vallon Volker. Sglt2 inhibition in a kidney with reduced nephron number: modeling and analysis of solute transport and metabolism. American Journal of Physiology-Renal Physiology 314(5): F969-F984, 2018.

[34] Layton Anita T, Vallon Volker, and Edwards Aurélie. Modeling oxygen consumption in the proximal tubule: effects of nhe and sglt2 inhibition. American Journal of PhysiologyRenal Physiology 308(12): F1343-F1357, 2015.

[35] Layton Anita T, Vallon Volker, and Edwards Aurélie. A computational model for simulating solute transport and oxygen consumption along the nephrons. American Journal of Physiology-Renal Physiology 311(6): F1378-F1390, 2016.

[36] Layton Anita T, Vallon Volker, and Edwards Aurélie. Predicted consequences of diabetes and sglt inhibition on transport and oxygen consumption along a rat nephron. American Journal of Physiology-Renal Physiology 310(11): F1269-F1283, 2016.

[37] Lee Way S and Sokol Ronald J. 2007, p. 259.

[38] Li Qianyi, McDonough Alicia A, Layton Harold E, and Layton Anita T. Functional implications of sexual dimorphism of transporter patterns along the rat proximal tubule: modeling and analysis. American Journal of Physiology-Renal Physiology 315(3): F692F700, 2018.

[39] Mik Egbert G, Johannes Tanja, Zuurbier Coert J, Heinen Andre, Houben-Weerts Judith HPM, Balestra Gianmarco M, Stap Jan, Beek Johan F, and Ince Can. In vivo mitochondrial oxygen tension measured by a delayed fluorescence lifetime technique. Biophysical Journal 95(8): 3977-3990, 2008.

[40] Mollica Maria P, Iossa Susanna, Liverini Giovanna, and Soboll Sibylle. Steady state changes in mitochondrial electrical potential and proton gradient in perfused liver from rats fed a high fat diet. Molecular and cellular biochemistry 178(1): 213-217, 1998. 
[41] Montalvo-Jave Eduardo E, Escalante-Tattersfield Tomas, Ortega-Salgado Jose A, Piña Enrique, and Geller David A. Factors in the pathophysiology of the liver ischemiareperfusion injury. Journal of Surgical Research 147(1): 153-159, 2008.

[42] Nagarajan Shilpa R, Paul-Heng Moumita, Krycer James R, Fazakerley Daniel J, Sharland Alexandra F, and Hoy Andrew J. Lipid and glucose metabolism in hepatocyte cell lines and primary mouse hepatocytes: a comprehensive resource for in vitro studies of hepatic metabolism. American Journal of Physiology-Endocrinology and Metabolism 316(4): E578-E589, 2019.

[43] Nastos Constantinos, Kalimeris Konstantinos, Papoutsidakis Nikolaos, Tasoulis MariosKonstantinos, Lykoudis Panagis M, Theodoraki Kassiani, Nastou Despoina, Smyrniotis Vassilios, and Arkadopoulos Nikolaos. Global consequences of liver ischemia/reperfusion injury. Oxidative medicine and cellular longevity 2014, 2014.

[44] Palmeira Carlos Marques, Teodoro João Soeiro, Amorim João Alves, Steegborn Clemens, Sinclair David A, and Rolo Anabela Pinto. Mitohormesis and metabolic health: The interplay between ros, camp and sirtuins. Free Radical Biology and Medicine 141: 483491, 2019.

[45] Petit Patrice X, O'Connor José E, Grunwald Didier, and Brown Spencer C. Analysis of the membrane potential of rat-and mouse-liver mitochondria by flow cytometry and possible applications. European journal of biochemistry 194(2): 389-397, 1990.

[46] Porter Richard K and Brand Martin D. Causes of differences in respiration rate of hepatocytes from mammals of different body mass. American Journal of PhysiologyRegulatory, Integrative and Comparative Physiology 269(5): R1213-R1224, 1995.

[47] Sato Nobuhiro, Kamada Takenobu, Kawano Sunao, Hayashi Norio, Kishida Yutaka, Meren Haruya, Yoshihara Harumasa, and Abe Hiroshi. Effect of acute and chronic ethanol consumption on hepatic tissue oxygen tension in rats. Pharmacology Biochemistry and Behavior 18: 443-447, 1983.

[48] Schönfeld Peter, Wiêckowski Mariusz R, and Wojtczak Lech. Thyroid hormone-induced expression of the adp/atp carrier and its effect on fatty acid-induced uncoupling of oxidative phosphorylation. FEBS letters 416(1): 19-22, 1997.

[49] Schwenke WD, Soboll S, Seitz HJ, and Sies H. Mitochondrial and cytosolic atp/adp ratios in rat liver in vivo. Biochemical Journal 200(2): 405-408, 1981.

[50] Sgouralis Ioannis, Evans Roger G, Gardiner Bruce S, Smith Julian A, Fry Brendan C, and Layton Anita T. Renal hemodynamics, function, and oxygenation during cardiac surgery performed on cardiopulmonary bypass: a modeling study. Physiological reports 3(1): e12260, 2015. 
[51] Sgouralis Ioannis, Evans Roger G, and Layton Anita T. Renal medullary and urinary oxygen tension during cardiopulmonary bypass in the rat. Mathematical medicine and biology: a journal of the IMA 34(3): 313-333, 2017.

[52] Soboll Sibylle, Elbers Rembert, Scholz Roland, and Heldt Hans-Walter. Subcellular distribution of di-and tricarboxylates and ph gradients in perfused rat liver. HoppeSeyler's Zeitschrift fur physiologische Chemie 361(1): 69-76, 1980.

[53] Somlyo Andrew P, Somlyo Avril V, and Shuman Henry. Electron probe analysis of vascular smooth muscle. composition of mitochondria, nuclei, and cytoplasm. The Journal of cell biology 81(2): 316-335, 1979.

[54] Sun SQ, Shi S-L, Hunt JA, and Leapman RD. Quantitative water mapping of cryosectioned cells by electron energy-loss spectroscopy. Journal of microscopy 177(1): 18-30, 1995.

[55] Suzuki Tomohiro, Shinjo Satoko, Arai Takatomo, Kanai Mai, and Goda Nobuhito. Hypoxia and fatty liver. World Journal of Gastroenterology: WJG 20(41): 15087, 2014.

[56] Valle Adamo, Guevara Rocio, Garcia-Palmer Francisco J, Roca Pilar, and Oliver J. Sexual dimorphism in liver mitochondrial oxidative capacity is conserved under caloric restriction conditions. American Journal of Physiology-Cell Physiology 293(4): C1302C1308, 2007.

[57] Wang Kening and Wondergem Robert. Hepatocyte water volume and potassium activity during hypotonic stress. The Journal of membrane biology 135(2): 137-144, 1993.

[58] Watanabe Fusao, Kamiike Wataru, Nishimura Tadashi, Hashimoto Tadao, and Tagawa Kunio. Decrease in mitochondrial levels of adenine nucleotides and concomitant mitochondrial dysfunction in ischemic rat liver. The Journal of Biochemistry 94(2): 493-499, 1983.

[59] Wojtczak Lech and Schönfeld Peter. Effect of fatty acids on energy coupling processes in mitochondria. Biochimica et Biophysica Acta (BBA)-Bioenergetics 1183(1): 41-57, 1993.

[60] Wu Fan, Yang Feng, Vinnakota Kalyan C, and Beard Daniel A. Computer modeling of mitochondrial tricarboxylic acid cycle, oxidative phosphorylation, metabolite transport, and electrophysiology. Journal of Biological Chemistry 282(34): 24525-24537, 2007.

[61] Yamashina Shunhei, Sato Nobuhiro, Kon Kazuyoshi, Ikejima Kenichi, and Watanabe Sumio. Role of mitochondria in liver pathophysiology. Drug Discovery Today: Disease Mechanisms 6(1-4): e25-e30, 2009.

[62] Zager Richard A. Mitochondrial free radical production induces lipid peroxidation during myohemoglobinuria. Kidney international 49(3): 741-751, 1996. 
[63] Zazueta Cecilia, Buelna-Chontal Mabel, Macías-López Arturo, Román-Anguiano Nadia G, González-Pacheco Héctor, Pavón Natalia, Springall Rashidi, ArandaFrausto Alberto, Bojalil Rafael, Silva-Palacios Alejandro, and others . Cytidine-5'diphosphocholine protects the liver from ischemia/reperfusion injury preserving mitochondrial function and reducing oxidative stress. Liver Transplantation 24(8): 1070$1083,2018$. 